\title{
Analysis of Influence Factors of Contemporary College Students' Political Identity Crisis
}

\author{
Xu Qiujie ${ }^{1, a,{ }^{*}}$, Zhang Xiurong ${ }^{2, b}$
}

${ }^{1}$ China University of Geosciences (Beijing) Marxist theory, Haidian district, Beijing, China ${ }^{2}$ China University of Geosciences (Beijing) institute of ideological and political education, Haidian district, Beijing, China

a272738154@qq.com, bzhxiur@sina.com

*Corresponding Author

\begin{abstract}
College students' political identification plays an critical role in China's political socialization, which is directly related to China's political stability and smooth modernization construction. Basically speaking, Contemporary college students' political identity tone is positive and healthy, but there are some crisis issue appeared in recent years. The problem including the students' instability inpolitical belief;questioning about the party and the government efficiency in dealing with social affairs ; false and utilitarian political identity, etc., which has inseparable relation with the social unfair phenomenon inour country, multiplepolitical corruptions, Western democratic thoughts and the tendency of practice to benefit of market economy, and the students' personal psychological factors. The exploring on the mechanism of influential factors has important significance to guide the benign development of the college students' political identity.
\end{abstract}

Keywords:College student, political identity ,crisis, influence factor.

\section{当代大学生政治认同危机影响因素分析}

\author{
许秋婕 ${ }^{1, \mathrm{a}, *}$ 张秀荣 ${ }^{2, \mathrm{~b}}$ \\ ${ }^{1}$ 中国地质大学 (北京) 马克思主义理论专业, 海淀, 北京, 中国 \\ 2 中国地质大学 (北京) 思想政治教育学院, 海淀, 北京, 中国 \\ a272738154@qq.com, 'zhxiur@sina.com \\ 通讯作者
}

中文摘要：大学生政治认同是我国政治社会 化命题中应有之要义, 直接关系到我国政治 稳定和现代化建设的顺利进行。现代大学生 政治认同基调是积极健康的，但也现出了政 治信仰动摇、质疑党和政府效能以及虚假功 利性的政治认同等危机状况，这与我国社会 不公平现象、政治腐败多发、西方民主思潮
以及市场经济的趋利性和大学生自身心理等 因素影响密不可分。探究影响因素的作用机 理, 对引导大学生政治认同良性发展有重要 意义。

关键词: 大学生 政治认同 危机 影响因素 


\section{1. 引言}

政治认同是认同在政治领域的反映, 是 “人们在社会政治生活中产生一种感情上的 归属感, 它与人们的心理活动有密切的关系。” 一般认为是政治学家罗森堡姆首先对政治认 同做出定义: “政治认同是社会认同的一部 分, 主要是指一个人在某些重要的主观意识 上对他所在的国家、民族、城镇居民、区域 等政治单位地理区域或团体的感觉。那些个 体感觉要强烈效忠、尽义务或责任的单位或 团体也属于政治认同的范畴。”大学生政治 认同就是指大学生在心理层面, 在个人情感 和意识上, 对现存的政治体系和社会政治生 活产生一种强烈的认同和归属感。

\section{2.当代大学生政治认同危机表现}

大学生政治认同的形成, 首先要求大学 生充分了解我国政治体系, 奠定良好的认知 基础, 由政治认知上升为对中国特色社会主 义的心理认同和归属, 并积极参与到政治活 动的实践中来。具体言之, 就是对中国特色 社会主义道路、理论体系和制度的最大自信、 支持和认同，积极参与社会主义现代化建设。 在现阶段, 大学生的政治认同主旋律是积极 向上的, 但由于国内、国际形势的深刻转变, 大学生的人生观、价值观面临多元化选择。 复杂的客观环境以及自身因素导致大学生在 政治认同上出现了不和谐的声音, 危机逐步 显现。

\section{1 政治价值认同危机}

政治价值认同危机主要表现在信仰上的 动摇和迷茫。信仰的认同是对政府或政党的 理念、信仰、目标和主张给予认可和信任, 是对马克思列宁主义、毛泽东思想和中国特 色社会主义理论体系以及中国特色社会主义 核心价值观的认同，即是对政治价值的认同。

当前绝大多数学生信仰马克思主义理论, 对中国特色社会主义 “一面旗帜、一条道路 和一个理论体系” 高度认同。在我国社会主 义市场经济体制下，不同的利益主体选取不 同的价值评判标准以期维护和谋取自身利益, 使得当前大学生面临价值多样化的选择。同 时, 各种各样的西方思潮潮水般涌入, 大学生 通过各种渠道, 例如网络能够获得直接的、
未经过滤的信息，五花八门丰富的信息猛烈 的冲击大学生思维。但学校思想政治教育往 往与实际相脱离, 主流的意识形态容易以碎 片化知识的形式传授给学生, 大学生未能用 学到的知识充分的解释各类社会现象带给他 们的迷茫和困惑。客观上由于中国与西方发 达国家的差距, 部分大学生对主流意识形态 产生怀疑, 相当一部分人认为马克思主义太 过于遥远复杂, 进入新世纪面对新的发展情 况, 马克思主义已经不适用了, 也会像其他 思想般 “过时”了; 也有一种想法认为我国 现在走的并不是社会主义道路，对实现共产 主义不抱希望, 对社会和个人缺乏信心, 拜 金主义和个人主义盛行, 甚至有少数学生不 知道该信仰什么, 呈现信仰真空的状态。

\section{2 政治效能认同危机}

政治效能认同是指对政治性公共政策的 绩效, 即所带来的公平和效率程度的认同感 和归属感。单靠理想信念和政治领袖的个人 魅力已经不能让当代大学生找到对我国社会 建设的归属感和认同感，他们对社会公平、 政府效能和自身发展等方面投以更多的关注。 大学生对党和政府绩效的考量和质疑, 引发 政治效能认同危机。

大学生在日常生活中更多关注与他们息 息相关的社会问题，比如就业，伴随着市场 经济的发展和中国经济结构的调整, 我国结 构性失业不断增加。尽管政府针对就业问题 采取相应政策, 鼓励大学生自主创业等, 但 现实就业需求和经济发展所能提供的就业岗 位之间的矛盾仍未能得到有效调和。大学生 毕业后就业压力显而易见。当前严峻的就业 形势削弱了政府在大学生间的公信力, 使大 学生认为政府效能低下。党和政府是人民公 仆的形象因贪污腐败现象层出不穷等原因在 大学生心目中大打折扣。例如: 因为对经济 适用房制度实施的监管力度不够, 让经济适 用房政策成为谋取不正当利益的手段，这一 政策的提出偏离了本意, 滋生了腐败现象。 这在一定程度上使学生认为政府所采取的平 衡收入分配、完善社会保障等方面的许多措 施, 并不能够实现预期的目标, 这些问题的出 现令大学生这一群体对党和政府的信心下降, 出现信任危机。 


\section{3 虚假政治认同和功利性认同趋于常态}

政治认同的虚假性是指认同主体的政治 态度和政治行为背离的状态。大学生由于外 在的压力或对自身利益的诉求, 隐藏内心真 实政治倾向，他们在公开场合表达的政治意 愿或对某种政治活动的认同与其真实的政治 意识、想法背离, 这种认同是虚假和功利的。

随着社会的发展当代中国的政治认同也 发生相应变化。这种变化主要表现在: 主流 意识形态的统领作用弱化, 政治价值取向从 一元化向多元化过渡, 虚假政治认同正是主 流意识形态弱化的一种表现。大学生政治认 同带有虚假性和功利性的现象屡见不鲜, 大 学生公开的政治态度与实际的行动之间差别 较大甚至完全相反, 在实际利益的驱动下, 大学生公共生活和私人生活中存在着两套话 语系统、两种语境甚至两种政治态度, 内心 真正想法与表达出的政治观点不符, “毕福 剑现象”已不是个例。大学生的虚假认同在 具有隐蔽性的网络上表现尤为突出。例如大 学生利用网络针对 7 - 23 温州动车追尾事故 的官员讲话内容, 匿名发表真实的“仇官” 言论和态度。但在入党的环节或在政治课堂 上的表现, 则表现出来更多的是对主流意识 形态的支持, 自己真正的政治态度已经被刻 意隐瞒。是什么原因导致大学生政治心理与 言行背离? 正是由于部分大学生的政治表达 是以利益为主导的, 事不关已便高高挂起, 政治冷漠是虚假政治认同的直接表现, 这种 冷漠和疏离是社会稳定与党的执政基础的隐 患。

\section{3. 大学生政治认同的影响因素探析}

马克思在曾指出: “不是人的社会意识决 定社会存在, 而是社会存在决定人们的精神 生活和政治生活领域。”大学生的政治认同 离不开国内外政治、经济、文化及自身因素 等的共同作用。

\section{1 转型期社会不公平撕裂了传统价值的认} 同

我国社会转型逐步推进, 社会阶层结构 变动、利益主体多元化, 使利益资源分割方 式和原有利益分配格局发生从高度的整体性、 一元向个体化、多元化转变。“人们奋斗所
争取的一切, 都同他们的利益有关。”伴随着 社会利益分化产生的就是社会公平问题, 公 平是价值范畴最高层面。社会公平就是在全 体社会成员之间公平合理的对社会的政治、 经济等利益进行分配。

收入分配不公平, 贫富差距扩大和发展 机会不公平是我国社会不公平现象的主要表 现。收入公平是社会公平的核心, 但由于区 域发展不平衡, 城乡发展不平衡, 人们之间 的收入差距也在不断扩大, 形成 “两头大, 中间小”的贫富格局。差距过大在某种程度 上是不公平原因造成的。美国科学史研究者 罗伯特 - 莫顿提出了著名的 “马太效应”, 即“任何个体、群体或地区，一旦在某个方面 如金钱、名誉、地位等获得成功和进步, 就会 产生一种积累优势, 会有更多的机会取得更 大的成功和进步。”贫富差距的拉大在极大 程度上造成了社会地位的悬殊, 从而导致富 人更富, 穷人更穷。在发展社会主义市场经济 的背景下，由于新旧体制交替和摩擦以及制 度上的漏洞催生了发展机会不公平问题, 例 如教育机会不公平问题, 目前我国东部与西 部、城市与农村在教育水平、教学资源等方 面有较大差异; 家庭背景、户口身份对教育 机会的影响力明显增强, 农民工子弟在城市 仍普遍存在入学难的现象。

大学生对与他们关心的问题寄于了更多 公正、平等、共同富裕的期待。社会发展差 距过大, 大量不公正、不合理的现象存在, 同中国共产党 “全心全意” 为人民服务的宗 旨、同 “像雷锋同志学习” 的社会精神、同 “共同富裕” 的奋斗目标背道而驰, 现实社 会不公平现象引起大学生心理落差, 导致大 学生政治认同刚性危机。主流意识形态共识 在大学生中产生极大的消解, 滋生冷漠、不 满、对立、仇视情绪, 不公平现象容易使大学 生产生心里鸿沟, 削弱凝聚力和向心力, 影响 社会稳定。

\section{2 西方民主思潮冲击导致对主流意识形态 认同的下降}

现代全球化的进程不断推进, 我国社会 转型日益深入, 我们已经进入了一个文化多 元的时代。我国始终坚持指导思想一元化与 思想文化多样性并存，有关民主思潮的种种 观念日渐进入大学生视野, 例如民主社会主 
义思潮在大学生之间有着较为深远影响。民 主思潮有利于大学生丰富知识, 开拓眼界, 但同时民主思潮在传播过程中对我国的主流 意识形态造成冲击。大学生盲目崇拜西方民 主思潮, 主要是由于民主思潮下的 “福利社 会” 建设契合了大学生的发展需求。瑞典所 实行的社会福利制度在某种程度上基本上实 现了共同富裕, 从摇篮到坟墓有全面的社会 保障及相关福利待遇, 这对于大学生有极大 的吸引力。大学生对于西方民主思潮中所倡 导的 “民主”、“自由” 和 “平等” 的概念 理解并不透彻, 与我国所提倡的民主概念混 淆, 存在盲目信从。例如民主社会主义思潮 在指导思想上多元化, 推崇多党制, 反对一 党制和无产阶级专政, 在社会发展道路上, 主张改良, 在理论和实践上注重功利。民主 社会主义披着 “民主” 和 “社会主义” 的外 套, 以为是民主和社会主义的结合。但其实 质依然是资产阶级民主, 根本上是为了维护 资产阶级的利益。

西方民主思潮宣扬 “社会主义意识形态 过时论”，中国与西方发达资本主义国家综 合实力客观上的差距, 让大学生开始怀疑马 克思主义理论已经失去时效, 使大学生错误 以为马克思主义 “无用”、已经破产; 同时, 西方民主思潮宣扬 自己走的是 “第三条道 路”, 即在社会主义和资本主义之外的选择, 大学生相信 “第三条道路” 存在的合理性。 但大学生未能立足于我国的实际国情, 认清 资本主义社会的一些新变化正是由于 “执行” 了马克思主义理论导致, 而使马克思主义的 基本原理更具有了客观基础。我国主流意识 形态的认同面临冲击和严峻挑战。

\section{3 政治腐败多发导致对政治效能认同的下}

\section{降}

政治腐败是指行使公共权力的公职人员 利用公共权力, 为了满足私人利益交换或占 有社会财富，导致公共利益受损。政府官员 清正廉洁是国家政治清明高效的缩影, 有利 于在大学生心目中树立良好的政治印象。胡 锦涛同志在 “七一” 讲话中指出, 我党现在 仍然面临精神懈急、能力不足、脱离群众和 消极腐败 “四个危险” 的严峻考验。每一个 执政党都会面临精神懈急的危险，使党内形 成消极政治生态；随着改革开放逐步深入，
国内经济的不平衡与国外经济危机的影响都 给党的执政能力、学习能力带来考验; 党的 兴亡系在始终保持和发展同人民群众的血肉 联系上, 但由于消极腐败现象的滋生, 损害 了党群关系, 直接影响我党执政基础和执政 合法性。

目前我国政治腐败现象多发，多表现在 用人腐败, 任人唯亲, 以不正当手段谋取、 巩固手中的权利; 司法腐败, 人治色彩浓厚 导致法律精神被茀视; 贪污贿赂之风蔓延。 这些腐败现象与大学生在日常教学中所学到 的关于党 “代表最广大人民的根本利益”, 政府是人民公仆的形象相差甚远，政府官员 不能做到勤政廉洁，那么行政的高效性就无 从谈起。无法达到大学生预期的高效型、服 务型政府的政治愿望, 政府官员也失去了表 率作用, 公众信任在逐渐丧失。腐败最致命的 危害是 “加重部分群体的相对剥夺感和受挫 感受, 进而造成了极个别的焦虑的人群、冷漠 的人群、误解的人群和越轨的人群”。“相 对剥夺感” 是大学生通过与自己地位相近的 个体或群体，比较彼此之间的生活状态，便 会产生的心理落差, 由此引发不信任感。从而 对党的执政认同不断消解，政治信任不断下 降，造成大学生的政治认同危机。

\section{4 市场经济的趋利性诱发政治认同的功利 化}

政治信仰是建立在社会物质条件的基础 上, 会以其为依据进行动态变化。追求利益 最大化是市场主体的行为目的, 市场经济的 效率主要来自于它的竞争性和开放性, 即通 过竞争来实现社会资源的最佳配置, 利益成 为了市场经济中最有力的机制。

市场经济以追求利益最大化为原则, 市 场竞争的基本机制反映到校园中, 就表现为 大学竞争意识强烈。但由于受到政治腐败、 投机取巧社会现象的影响, 使得小团体主义、 利已主义、个人主义、拜金主义等价值观在 大学生中泛滥, 大学生在学习、入党和择业 观上多有虚假、功利化选择, 就入党动机而 言，大学生更多是因为党员身份更有利于毕 业找工作或者参加公务员考试，单纯把入党 看作竞争筹码, 而忽略了我党真正要求的全 心全意为人民服务的宗旨。他们更加关注眼 前的、物质上的利益, 对要求 “无私奉献” 的 
社会精神冷漠对待, 各人自扫门前雪, 对公 共性质的社会问题关注较少, 更多将权重放 在满足自身利益需求上。部分学生在政治价 值取向和政治行为上带有虚假性、功利性。

\section{5 大学生政治心理不成熟是影响政治认同 的重要因素}

影响大学生政治认同感的另一个重要因 素是大学生的政治心理不成熟。我国大学生 正是处于精力旺盛, 思维敏捷的青少年时期, 但也正因为如此, 大学生心理发展尚未成熟, 思考问题缺乏深刻和理性, 个人喜好是普遍 的判断标准。同时, 大学生接受较高的教育 水平使他们的公民主体意识和个人权利意识 不断增强, 又具有一定的独立和批判的思维, 对政治问题能够形成自己的判断和理解。这 使得大学生对我国的政治期待增加, 单纯依 靠理想主义、意识形态、个人鬼力等获取认 同的方式逐渐失效，对政治权威性认同逐渐 消解。大学生自身个人权利意识觉醒, 但政 治心理不够成熟的特点直接影响着他们的政 治认同的程度。

\section{4.结束语}

政治认同是政治稳定的重要前提，只有 从根本上提高大学生对中国特色社会主义的 理性认知, 深化大学生对中国政治的认同, 才能使他们在心理层次上形成对中国特色社 会主义道路、理论、制度的拥护，才能从根 本上调动他们的积极性与主动性, 对政治稳 定起到深度支撑的作用，促进社会的和谐发 展。

\section{References}

[1] Zhou Wuyi.Encyclopedia of China-Political Science [M]. Beijing: China encyclopedia publishing house, pp.500-501,2004.

[2] [US] Rosenbaum. Political Culture [M]. Taipei Crown Books Co. Ltd.pp.6,1984.

[3] Zhang Meihua. Exploration of Countermeasure and Present Situation on Political Identification of Contemporary University Student[J].Journal of Shayang
Teachers College, pp.28,2007.3

[4] Liang Aiqiang. The Construction of Political Identity of Young Students from the Perspective of Social Conflict Theory[J].Studies on the Socialism with Chinese Characteristics, pp.95,2014.5

[5] Selections of K. Marx and F. Engels. Vol.2 [M]. Beijing: People's Publishing House, pp.82,1972.

[6] Marx, Engels, Complete Works of Karl Marx and F. Engels (Vol.1). Beijing: People's Publishing House, pp.82,1956.

[7] Zhou Qing. Mattew Effect of the Archives Work in Colleges and Universities [J].Goobal Market Information Guide, 2011.4

[8] Wu Shinan. Corruption, the Decline of Public Social Psychological Factors in China [J]. Journal of Yanbian University, pp. 61-65,1999.1 